\title{
DECISIVE SUBGROUPS OF ANALYTIC GROUPS
}

BY

\author{
T. CHRISTINE STEVENS
}

\begin{abstract}
It is known that every analytic group $(L, \tau)$ contains a closed abelian subgroup $H$ which is "decisive" in the sense that the Hausdorff topologies for $L$ which are weaker than $\tau$ are completely determined by their restrictions to $H$. We show here that $H$ must ordinarily contain the entire center of $L$ but that the rest of $H$ can in general be reduced. The proof involves constructing "unusual" topologies for abelian Lie groups.
\end{abstract}

1. Introduction. A description of the ways in which the topology of an analytic group can be weakened while remaining Hausdorff is important to the study of arcwise-connected, finite-dimensional, metric groups and, more generally, to an understanding of immersions of analytic groups. In [8] it was shown that every analytic group $(L, \tau)$ contains a closed abelian subgroup $H$ which is "decisive" in the sense that the Hausdorff topologies for $L$ which are weaker than $\tau$ are completely determined by their restrictions to $H$. In the present paper we use a recent result of Goto [3] to refine the description of $H$ that is given in [8] and then proceed to the question of whether $H$ is the "smallest" decisive subgroup of $L$. Our answer, presented in $\S 3$, is that $H$ must ordinarily contain the entire center of $L$ but that the rest of $H$ can in general be reduced. The proof is contained in $\$ 5$ and relies upon a technical procedure for constructing "unusual" topologies for abelian Lie groups that comprises $\S 4$. These topologies turn out to be of interest in their own right, and a discussion of their significance appears in $\$ 6$.

2. Notation and terminology. Topologies for abstract groups are assumed to be Hausdorff and to make the group operations continuous. If $(G, \mathscr{U})$ is a topological group and $A$ is a subgroup of $G$, then $\mathscr{U}_{A}$ denotes the topology which $A$ inherits from $\mathcal{U}$, and $T(G, \mathcal{U})$ denotes the collection of all group topologies for $G$ which are weaker than $\mathscr{U}$. We will be interested in the following technique for extending topologies on $A$ to all of $G$. Let $Q$ be a group toplogy for $A$ which makes the function $f: G \times A \rightarrow G$, defined by $f(x, a)=a x a^{-1},(\mathscr{Q} \times \mathscr{Q}, \mathcal{Q})$-continuous. If $A$ is $\mathscr{Q}$-closed and $\mathcal{Q}$ is weaker than $\mathcal{U}_{A}$, then the collection

$$
\{P N: P \text { a } U \text {-neighborhood of } e, N \text { an } Q \text {-neighborhood of } e\}
$$

is the basis for a (Hausdorff) group topology for $G$ which is called the standard extension of $\mathcal{Q}$ to $G$, denoted $\mathcal{E}(\mathbb{Q})$. $\mathcal{E}(\mathbb{Q})$ may be characterized as the strongest

Received by the editors July 13, 1981; presented to the Society January 4, 1980.

1980 Mathematics Subject Classification. Primary 22E15; Secondary 22A05, 22E40.

Key words and phrases. Analytic group, Lie group, (CA) analytic group.

(C) 1982 American Mathematical Society 0002-9947/81/0000-0455/\$03.00 
topology for $G$ which is weaker than $\mathcal{Q}$ and makes the inclusion map $i:(A, \mathbb{Q}) \rightarrow G$ continuous. We say that $A$ is decisive in $G$ if $A$ is $\mathscr{Q}$-closed and if $\mathscr{V}=\mathcal{E}\left(\mathscr{V}_{A}\right)$ for every $\mathscr{V} \in T(G, \mathcal{Q})$. A more detailed discussion of these notions may be found in $\S 3$ of [8].

When $(G, \mathcal{Q})$ is a connected Lie group, there are special conditions which assure the continuity of the function $f$ mentioned above. Let $\mathbf{g}$ be the Lie algebra of $G$, $\mathrm{Gl}(\mathrm{g})$ the group of linear automorphisms of $\mathrm{g}$, and $\mathbb{Q}$ a topology for $A$. If the restriction of the adjoint representation, $\mathrm{Ad}: A \rightarrow \mathrm{Gl}(\mathrm{g})$, is Q fact that $\operatorname{Aut}(G)$ is isomorphic with a closed subgroup of $\mathrm{Gl}(\mathrm{g})$ implies that $f$ is continuous. In particular, by 4.2 in [8], $f$ is continuous if $Q=\mathscr{V}_{A}$, where $\mathscr{V} \in T(G, \mathcal{Q})$.

$\mathbf{Z}, \mathbf{R}, T^{q}$, and $\mathrm{Gl}(V)$ denote, respectively, the integers, the real numbers, the $q$-dimensional torus, and the group of linear automorphisms of a vector space $V$. The relative topology for a subgroup $A$ of $\mathrm{Gl}(V)$ is called the full-linear-group ( $f l g$ ) topology for $A$. The symbol $\square$ marks the end of a proof.

3. Main results. Our first theorem is a modification of the main Theorem (3.2) in [8].

3.1 THEOREM. Let $(L, \tau)$ be a connected Lie group with Lie algebra l.

(i) L contains an abelian decisive subgroup $H$ of the form $\mathbf{R}^{p} \times T^{q} \times \mathbf{Z}^{r} \times D$, where $p, q$, and $r$ are nonnegative integers and $D$ is finite. The adjoint image of $H$ is contained in a toroid $Q$.

(ii) The topologies in $T(L, \tau)$ are precisely those of the form $\mathcal{E}(\mathscr{B})$, where $\mathscr{B} \in$ $T\left(H, \tau_{H}\right)$ and the restriction of the adjoint representation, $\mathrm{Ad}: H \rightarrow Q$, is $\Re_{\text {-continu- }}$ ous.

(iii) If $(L, \tau)$ is (CA) - that is, if the adjoint image of $L$ is flg-closed in $\mathrm{Gl}(l)$-then $H$ is the center $Z(L)$ of $L$.

(iv) If $(L, \tau)$ is not (CA), then $H=V \times Z(L)$, where $V$ is a vector group.

Proof. Except for (iv), this theorem is identical to 3.2 in [8], which makes the more limited assertion that $H=V \times J$, where $J$ contains $Z(L)$ and $J / Z(L)$ is finite (trivial if $L$ is solvable). By indicating certain changes that can be made in the previous proof, we will show that, if $(L, \tau)$ is not (CA), then $H=V \times Z(L)$ whether $L$ is solvable or not. We denote by $\mathcal{Q}$ the connected Lie topology for $\operatorname{Ad}(L)$ and by $C$ the flg-closure of $\operatorname{Ad}(L)$. Let $N$ be a maximal $थ$-connected and flg-closed subgroup of $\operatorname{Ad}(L)$ which contains the commutator subgroup of $\operatorname{Ad}(L)$. The key to the new proof is that one may now, by virtue of $\$ 5$ of [3], find a torus $T$ in $C$ and a Q-closed vector subgroup $W$ of $\operatorname{Ad}(L)$ such that $\operatorname{Ad}(L)=N W, C=N T, T$ is the flg-closure of $W$, and $N \cap T$ is trivial (not just finite). For if $T_{1}$ is a maximal torus in $C$, then $N \cap T_{1}$ is connected (by 5.1 in [3]), and we can let $T$ be a torus in $T_{1}$ which is complementary to $N \cap T_{1}$. Since $C=\operatorname{Ad}(L) \cdot T$, (i), (ii) and (iii) follow as in $\S 4$ of [8]. To prove (iv), we note that Zerling's structure theorem [9] is compatible with this new decomposition $C=N T$. Thus 6.1 in [8] remains valid, and the argument which follows 6.1 and which shows that $H=V \times Z(L)$ now applies even without the hypothesis of solvability. 
From 3.1 we see that "unusual" topologies for abelian Lie groups deserve our attention. Although the fact that a compact topology cannot be weakened and remain Hausdorff tempts us to ignore the compact part of $H$, we will show in $\S 4$ that, unless $H$ is itself compact, there are topologies in $T\left(H, \tau_{H}\right)$ which involve both the compact and the noncompact parts of $H$. This will enable us to demonstrate the following theorem, the proof of which appears in $\$ 5$.

3.2 THEOREM. Let $(L, \tau)$ be a connected Lie group.

(i) $Z(L)$ is decisive in $L$ if and only if $(L, \tau)$ is (CA).

(ii) $T(L, \tau)$ is trivial-i.e., $T(L, \tau)$ consists of the single topology $\tau$-if and only if $(L, \tau)$ is $(\mathrm{CA})$ and $Z(L)$ is compact.

(iii) If $T(L, \tau)$ is not trivial, then $Z(L)$ is contained in every decisive subgroup of $L$. In particular, the compact part of $Z(L)$ cannot be eliminated.

(iv) If $(L, \tau)$ is not (CA) and $n$ is the dimension of the vector group $V$ in 3.1(iv), then $V$ contains a subgroup $Y$ which is $\tau$-isomorphic to $\mathbf{Z}^{n}$ such that $Y \times Z(L)$ is decisive in $L$.

4. Weakening the topology of an abelian Lie group. In this section we describe a procedure for constructing the "unusual" topologies for $\mathbf{R}^{n}$ that will be needed to prove Theorem 3.2. $\tau_{n}$ denotes the usual topology and \|\| the usual norm for $\mathbf{R}^{n}$. Our first result shows that any sequence $\left\{v_{j}\right\}$ in $\mathbf{R}^{n}$ which "goes to infinity sufficiently fast" in $\tau_{n}$ can be made to converge to zero in a topology in $T\left(\mathbf{R}^{n}, \tau_{n}\right)$. Somewhat similar results can be found in [6] and [7], but our work differs from Nienhuys' by allowing $n$ to be greater than 1 and by not requiring the sequence $\left\{v_{j}\right\}$ to be integral. Throughout this section, $N$ denotes the set of natural numbers, and we assume that sums contain only finitely many nonzero terms.

4.1 Proposition. Let $\left\{p_{j}: j \in N\right\}$ be a nonincreasing sequence of positive real numbers which converges to zero in $\tau_{1}$, and let $\left\{v_{j}: j \in N\right\}$ be a sequence of nonzero elements of $\mathbf{R}^{n}$ such that $\left\{\left\|v_{j}\right\|\right\}$ is nondecreasing and the sequence

$$
\left\{p_{j+1}\left\|v_{j+1}\right\| /\left\|v_{j}\right\|\right\}
$$

has a positive lower bound. Then the function $\nu: \mathbf{R}^{n} \rightarrow \mathbf{R}$ defined by

$$
\nu(x)=\inf \left\{\sum\left|c_{j}\right| p_{j}+\left\|x-\sum c_{j} v_{j}\right\|: c_{j} \in \mathbf{Z}\right\}
$$

is a norm on $\mathbf{R}^{n}$ such that $\nu(x) \leqslant\|x\|$ for all $x \in \mathbf{R}^{n}$ and $\nu\left(v_{j}\right) \leqslant p_{j}$. $\nu$ gives rise to a metrizable topology in $T\left(\mathbf{R}^{n}, \tau_{n}\right)$ in which $v_{j} \rightarrow 0$.

We begin the proof with a useful lemma which states that any nonzero vector which can be expressed as a linear combination for "large" $v_{j}$ 's has a large norm.

4.2 LEMMA. Let the hypotheses be as in 4.1. For every $k>0$, there is a $d>0$ such that, if $\Sigma\left|c_{j}\right| p_{j}<d$ and $c_{j} \in Z$, then either $c_{j}=0$ for all $j$ or $\left\|\sum c_{j} v_{j}\right\|>k$.

Proof. Let $r$ be a positive lower bound for the sequence (1). It is easy to see that there must exist a natural number $s$ such that $p_{s}<\frac{r}{2}$ and $p_{j+1} /\left\|v_{j}\right\|<\frac{r}{2 k}$ for all $j \geqslant s$. Let $d=p_{s}$, and suppose that $\Sigma\left|c_{j}\right| p_{j}<d,\left\|\Sigma c_{j} v_{j}\right\| \leqslant k$, and some $c_{j} \neq 0$. 
Letting $b=\sum c_{j} v_{j}$ and letting $M$ denote the largest integer $j$ such that $c_{j} \neq 0$, we see that $M>s$ and

$$
\begin{aligned}
\left|c_{M}\right|\left\|v_{M}\right\|-\|b\| & \leqslant\left\|c_{M} v_{M}-b\right\|=\left\|\sum_{j<M} c_{j} v_{j}\right\| \\
& \leqslant\left(\sum_{j<M}\left|c_{j}\right|\right)\left\|v_{M-1}\right\| .
\end{aligned}
$$

From (2) it follows that

$$
\sum_{j<M}\left|c_{j}\right| p_{j} \geqslant\left(\sum_{j<M}\left|c_{j}\right|\right) p_{M} \geqslant \frac{\left|c_{M}\right|\left\|v_{M}\right\| p_{M}}{\left\|v_{M-1}\right\|}-\frac{\|b\| p_{M}}{\left\|v_{M-1}\right\|} .
$$

Since the first term on the right side of (3) is at least $r$ and the second is less than $\frac{r}{2}$, we conclude that

$$
\sum\left|c_{j}\right| p_{j} \geqslant \sum_{j<M}\left|c_{j}\right| p_{j} \geqslant r-\frac{r}{2}>d
$$

This contradiction proves the lemma.

Proof of 4.1. The only part of the proposition which is not immediately clear is the fact that $\nu(x)=0$ only if $x=0$. To dispose of this detail, we suppose that $x \in \mathbf{R}^{n}$ is given and then apply 4.2 to obtain a $d>0$ corresponding to $k=\|x\|+1$. If $\nu(x)=0$, then for every $\varepsilon, 0<\varepsilon<\min (d, 1)$, there is an element $b=\Sigma c_{j} v_{j}$ of the subgroup generated by $\left\{v_{j}\right\}$ such that $\|x-b\|<\varepsilon<1$ and $\Sigma\left|c_{j}\right| p_{j}<\varepsilon<d$. Since $\|b\| \leqslant\|x\|+1=k$, it follows form 4.2 that $b=0$. Therefore $\|x\|<\varepsilon$ for arbitrary positive $\varepsilon$, and thus $x=0$.

If $\left\{v_{j}\right\}$ and $\left\{p_{j}\right\}$ satisfy the hypotheses of 4.1 , we will call $\left\{v_{j}\right\}$ and $\left\{p_{j}\right\}$ a norming pair and $\left(\left\{v_{j}\right\},\left\{p_{j}\right\}, v\right)$ a norming triple for $\mathbf{R}^{n}$. One easily verifies, for example, that $\{n !+1\}$ and $\left\{\frac{1}{n}\right\}$ form a norming pair for $\mathbf{R}$. The following corollary to 4.1 identifies certain $\left\{v_{j}\right\}$ which "go to infinity" fast enough to be part of a norming pair.

4.3 Corollary. If $\left\{v_{j}: j \in N\right\}$ is a sequence of nonzero elements of $\mathbf{R}^{n}$ such that $\left\{\left\|v_{j+1}\right\| /\left\|v_{j}\right\|\right\}$ monotonically increases to $+\infty$, then there is a metrizable topology in $T\left(\mathbf{R}^{n}, \tau_{n}\right)$ in which $v_{j} \rightarrow 0$.

Proof. Let $p_{1}=1$ and, for $j \geqslant 1$, let $p_{j+1}=\left\|v_{j}\right\| /\left\|v_{j+1}\right\|$. Since we may assume that $\left\|v_{j+1}\right\| \geqslant\left\|v_{j}\right\|$ for all $j$, it is easy to verify that $\left\{v_{j}\right\}$ and $\left\{p_{j}\right\}$ form a norming pair.

We now consider a procedure for obtaining a norming pair for $\mathbf{R}^{n+1}$ from one for $\mathbf{R}^{n}$. In this discussion it will sometimes be convenient to write an element of $\mathbf{R}^{n+1}$ in the form $(a, b)$, where $a \in \mathbf{R}^{n}$ and $b \in \mathbf{R}$. If $\left(\left\{v_{j}\right\},\left\{p_{j}\right\}, v\right)$ is a norming triple for $\mathbf{R}^{n}$ and $y_{j}=0$ for all $j \in N$, then $\left\{\left(v_{j}, y_{j}\right)\right\}$ and $\left\{p_{j}\right\}$ clearly form a norming pair for $\mathbf{R}^{n+1}$, and the associated topology for $\mathbf{R}^{n+1}$ is simply the product of the $\nu$-topology for $\mathbf{R}^{n}$ and the usual topology for $\mathbf{R}$. A different choice of the sequence $\left\{y_{j}\right\}$, however, will "twist" the $\nu$-topology in such a way that the projections of $\mathbf{R}^{n+1}$ onto $\mathbf{R}^{n} \times 0$ and $0 \times \mathbf{R}$ need not be continuous. The next proposition describes a scheme for choosing $\left\{y_{j}\right\}$. 
4.4 Proposition. Let $\left(\left\{v_{j}\right\},\left\{p_{j}\right\}, \nu\right)$ be a norming triple for $\mathbf{R}^{n}$, and let $\left\{y_{j}\right\}$ be a sequence in $\mathbf{R}$ such that $\left|y_{j}\right| \leqslant \min \left(\left|y_{j+1}\right|,\left\|v_{j}\right\|\right)$ for all $j \in N$. Let $w_{j}=\left(v_{j}, y_{j}\right)$.

(i) $\left\{w_{j}\right\}$ and $\left\{p_{j}\right\}$ are a norming pair for $\mathbf{R}^{n+1}$.

(ii) If $\mu$ is the norm associated with $\left\{w_{j}\right\}$ and $\left\{p_{j}\right\}$, then $\nu(x) \leqslant \mu(x, z)$ for every $x \in \mathbf{R}^{n}, z \in \mathbf{R}$.

(iii) $0 \times \mathbf{R}$ (the last factor of $\mathbf{R}^{n+1}$ ) inherits the usual topology from the $\mu$-topology.

Proof. The inequality

$$
\frac{\left\|\left(v_{j+1}, y_{j+1}\right)\right\|}{\left\|\left(v_{j}, y_{j}\right)\right\|} \geqslant \frac{\left\|v_{j+1}\right\|}{2\left\|v_{j}\right\|}
$$

implies that $\left\{w_{j}\right\}$ and $\left\{p_{j}\right\}$ satisfy the hypotheses of 4.1 , and thus (i) is proved. Part (ii) is clear from the definitions of $\nu$ and $\mu$. We will prove (iii) by showing that, for every $k>0$, there exists a $t>0$ such that $\|(0, z)\|<k$ if $\mu(0, z)<t$. Applying 4.2 to $\left\{v_{j}\right\}$ and $\left\{p_{j}\right\}$, we first obtain a $d>0$ corresponding to the given number $k$ and then let $t=\min (d, k)$. If $\mu(0, z)<t$, there is an element $(a, b)=\Sigma c_{j}\left(v_{j}, y_{j}\right), c_{j} \in \mathbf{Z}$, of the subgroup generated by $\left\{w_{j}\right\}$ such that $\|(0, z)-(a, b)\|<t \leqslant k$ and $\Sigma\left|c_{j}\right| p_{j}<t$ $\leqslant d$. It follows that $a=\Sigma c_{j} v_{j}$ has norm less than $k$ (in the usual norm for $\mathbf{R}^{n}$ ), and by 4.2 every $c_{j}$ equals zero. Therefore $(a, b)=(0,0)$, and $\|(0, z)\|<k$.

From 4.4(iii) we see that the projection of $\left(\mathbf{R}^{n+1}, \mu\right)$ onto $0 \times \mathbf{R}$ (with the relative topology) is continuous if and only if $\left|y_{j}\right| \rightarrow 0$. For example $\left(\left\{\left(n !+1, \frac{\pi}{2}\right)\right\},\left\{\frac{1}{n}\right\}\right)$ is a norming pair that induces a "nonproduct" topology on $\mathbf{R}^{2}$, in the sense that the projection of $\mathbf{R}^{2}$ onto $(0 \times \mathbf{R}, \mu)$ is not continuous. The significance of such topologies here is that they enable us to construct topologies for $\mathbf{R}^{n} \times T^{1}$ which twist the compact, as well as the noncompact, factor. The next proposition shows how to do this.

4.5 Proposition. Let the notation be as in 4.4, and let $\mathscr{Q}$ denote the usual topology for $\mathbf{R}^{n} \times T^{1}$, which is algebraically isomorphic to $\mathbf{R}^{n+1}(0 \times \mathbf{Z})$. Then the topology of which $\mu$ induces on $\mathbf{R}^{n+1} /(0 \times \mathbf{Z})$ is in $T\left(\mathbf{R}^{n} \times T^{1}, \mathcal{Q}\right)$, and $\left(v_{j}, y_{j} \bmod Z\right) \rightarrow e$ in $\mho$. Moreover, if $\pi: \mathbf{R}^{n} \times T^{1} \rightarrow \mathbf{R}^{n}$ is the natural projection and $\chi: \mathbf{R}^{n} \rightarrow T^{1}$ is a v-continuous homomorphism, then $\chi \circ \pi: \mathbf{R}^{n} \times T^{1} \rightarrow T^{1}$ is W-continuous.

Proof. Since $0 \times \mathbf{Z}$ is $\mu$-discrete by 4.4 (iii), everything except the last sentence of 4.5 is obvious. To prove that $\chi \circ \pi$ is $\mathscr{W}$-continuous, it suffices to observe that 4.4(ii) assures the continuity of the natural projection of $\left(\mathbf{R}^{n+1}, \mu\right)$ onto $\left(\mathbf{R}^{n}, \nu\right)$, the kernel of which contains $0 \times \mathbf{Z}$.

Although 4.5 refers explicitly only to $\mathbf{R}^{n} \times T^{1}$, we note here that appropriate choice of $\left\{v_{j}\right\}$ and $\left\{y_{j}\right\}$, followed by restriction of the resulting topology, produces similar topologies for $\mathbf{Z}^{n} \times T^{1}$ and for the product of $\mathbf{R}^{n}$ or $\mathbf{Z}^{n}$ with a finite group.

5. Proof of 3.2. In this section we will use the topologies constructed in $\$ 4$ to prove Theorem 3.2, but first we make some general observations about decisive subgroups. Let $(L, \tau)$ be an analytic group, let $\mathcal{Q} \in T(L, \tau)$, and let $\left\{x_{m}\right\}$ be a net in $L$ which Q-converges to $e$. We claim that, if $J$ is a decisive subgroup of $L$, then $\left\{x_{m}\right\}$ has a subnet $\left\{x_{m(i)}\right\}$ which can be written in the form $x_{m(i)}=a_{i} j_{i}$, where $\left\{a_{i}\right\}$ is a net in $L$ 
which $\tau$-converges to $e$ and $\left\{j_{i}\right\}$ is a net in $J$ which $\mathcal{Q}_{J}$-converges to $e$. For $J$ acts on $L$ by inner automorphism and, denoting by $L \times_{I} J$ the corresponding semidirect product, we can define a homomorphism $f: L \times{ }_{I} J \rightarrow L$ by $f(x, y)=x y$. The fact that $J$ is decisive in $L$ means that $f$ is $\left(\tau \times \mathscr{Q}_{J}, \mathscr{Q}\right)$-open and continuous, and the claim follows. Many of the nets which will arise in the proof of 3.2 are contained in the normalizer $N$ of $J$, and thus it is useful to rephrase our claim as follows.

5.1 Lemma. Let $J$ be decisive in the analytic group $(L, \tau)$, and let $N$ be the normalizer of $J$ in $L$, with $\pi: N \rightarrow N / J$ the natural projection and $\mathcal{K}$ the quotient topology for $N / J$ from $\tau$. If $थ \in T(L, \tau)$ and $\left\{x_{m}\right\}$ is a net in $N$ which W-converges to $e$, then $\left\{x_{m}\right\}$ has a subnet $\left\{x_{m(i)}\right\}$ such that $\pi\left(x_{m(i)}\right) \rightarrow e$ in $\Re$.

We proceed now to the proof of 3.2, during which $l$ will denote the Lie algebra of $L$. The $(\Leftarrow)$ part of $3.2(\mathrm{i})$ is $3.1(\mathrm{iii})$; to prove $(\Rightarrow)$, suppose that $Z(L)$ is decisive in $L$ and $(L, \tau)$ is not (CA). Then the connected Lie topology $\mathscr{T}$ for $\operatorname{Ad}(L)$ is strictly stronger than the flg topology, and thus $L$ contains a one-parameter subgroup $\lambda$ such that $\operatorname{Ad}(\lambda)$ is closed in $\mathscr{V}$ but not in flg. Since the adjoint representation must be injective on $\lambda$, we may regard the flg-topology as a topology for $\lambda$ and form its standard extension $\mathscr{Q}$ to $L$. Let $\left\{x_{m}\right\}$ be a net in $\lambda$ which flg-converges to $e$ but which has no subnet that $\mathcal{V}$-converges to $e$. Then $\mathscr{U} \in T(L, \tau), x_{m} \rightarrow e$ in $\mathcal{U}$, and we may apply 5.1 with $J=Z(L)$ and $N=L$ to obtain a subnet $\left\{x_{m(i)}\right\}$ such that $\pi\left(x_{m(i)}\right) \rightarrow e$ in $\mathscr{T}$. Since $(L / Z(L), \mathscr{T})$ is homeomorphic with $(\operatorname{Ad}(L), \mathscr{V})$, this contradicts the fact that $x_{m(i)} \nrightarrow e$ in $\mathscr{V}$ and completes the proof of 3.2(i).

Part (ii) of 3.2 follows from the theorem in [2]. Because the proof of 3.2(iii) will involve nonproduct topologies, we must first carefully establish our notation. As an abelian Lie group, $Z(L)$ has the form $\mathbf{R}^{a} \times T^{b} \times E$, where $a$ and $b$ are nonnegative integers and $E$ is discrete. By Theorem $1^{\prime}$ and the subsequent remark in [5], $E$ is finitely generated, and thus we can write $Z(L)$ as $\mathbf{R}^{a} \times \mathbf{Z}^{r} \times T^{b} \times F$, where $a, b$, and $r$ are nonnegative integers and $F$ is finite. If $e_{1}$ and $e_{2}$ are, respectively, the identities of $\mathbf{R}^{a} \times \mathbf{Z}^{r}$ and $T^{b} \times F$, we will denote the compact part $e_{1} \times T^{b} \times F$ of $Z(L)$ by $C$ and $\mathbf{R}^{a} \times \mathbf{Z}^{r} \times e_{2}$ by $D$. Now let $J$ be a decisive subgroup of $L$. Clearly it will suffice to show that $J$ contains both $C$ and $D$. The proof, which uses the notation of 5.1 , is divided into three parts.

(1) $J$ contains $D$. Suppose $x \in D$ but $x \notin J$. Then there exist symmetric $\tau_{N}$-neighborhoods $W$ and $V$ of $e$ such that $\pi(x) \notin \pi(V)$ and $W^{2} \subseteq V$. Since $\pi(x) \in \pi(V)$ if both $\pi\left(x^{n}\right)$ and $\pi\left(x^{n+1}\right)$ are in $\pi(W)$, there are arbitrarily large integers $n$ such that $\pi\left(x^{n}\right) \notin \pi(W)$. Now $x$ generates a subgroup $A$ of $Z(L)$ which is isomorphic to $\mathbf{Z}$, and from $A$ we can choose a sequence $\left\{x^{n(i)}: i\right.$ a positive integer $\}$ satisfying the hypotheses of 4.3 and such that $\pi\left(x^{n(i)}\right) \notin \pi(W)$ for all $i$. If $Q$ is the restriction to $A$ of the corresponding topology for $\mathbf{R}$, then $\mathcal{E}(\mathbb{Q})$ is in $T(L, \tau), x^{n(i)} \rightarrow e$ in $\mathcal{E}(\mathbb{Q})$, and it follows from 5.1 that some subnet of $\left\{\pi\left(x^{n(i)}\right)\right\}$ converges to $e$ in $\Re$. This contradicts the fact that $\pi\left(x^{n(i)}\right) \notin \pi(W)$ for all $i$, and thus $x \in J$.

(2) $J$ contains $C$ if $Z(L)$ is not $\tau$-compact. Since $J$ is $\tau$-closed, it suffices to show that $J$ contains every element of $C$ of finite order. If $y$ is such an element and $x$ is a nontrivial element of $D$, we may identify the subgroup generated by $x$ and $y$ with 
$\mathbf{Z} \times E$, where $E$ is finite. Using 4.3, 4.4, and 4.5, we can find a sequence $\left\{x^{n(i)}: i\right.$ a positive integer $\}$ and topologies $\mathcal{Q}$ for $\mathbf{Z}$ and $\mathcal{V}$ for $\mathbf{Z} \times E$ such that $x^{n(i)} \rightarrow e$ in $\mathscr{Q}$ and $x^{n(i)} y \rightarrow e$ in $\mathcal{V}$. Applying 5.1 to $\mathcal{E}(\mathscr{Q})$ and $\mathcal{E}(\mathcal{V})$, we obtain a subnet $\left\{x^{n\left(i_{k}\right)}\right\}$ such that both $\left\{\pi\left(x^{n\left(i_{k}\right)}\right)\right\}$ and $\left\{\pi\left(x^{n\left(i_{k}\right)} y\right)\right\}$ converge to the identity in $\Re$. Therefore $\pi(y) \rightarrow e$ in $\Re$, and thus $y \in J$.

(3) $J$ contains $C$ if $Z(L)$ is $\tau$-compact. Again we let $y$ be an element of $C$ of finite order, but the fact that $D$ is trivial means that we cannot choose $\left\{x^{n(i)}\right\}$ as in (2). The hypothesis that $T(L, \tau)$ is not trivial combines with 3.2(ii) to assure that $(L, \tau)$ is not (CA), and thus we can let $\lambda$ and $\mathcal{Q}$ be as in the proof of (i). If $E$ is the group generated by $y$, then $\lambda E$ is easily seen to be $\tau$-isomorphic to $\mathbf{R} \times E$, since $\lambda$ has closed image in $L / Z(L)$ in the quotient topology from $\tau$. By 4.3, 4.4, and 4.5, there exist a sequence $\left\{x_{i}\right\}$ in $\lambda$ and a topology $\mathcal{Y}$ for $\lambda E$ such that $\operatorname{Ad}\left(x_{i}\right) \rightarrow e$ in the flg-topology and $x_{i} y \rightarrow e$ in $\mathcal{Y}$. Since $\operatorname{Ad}(\lambda)$ is contained in a torus, 4.5 also assures that Ad: $\lambda E \rightarrow \mathrm{Gl}(l)$ is $\mathcal{O}$-continuous, and this allows us to form $\mathscr{E}(\mathcal{Y})$. From the remarks preceding 5.1, applied to $\mathscr{U}$ and $\mathcal{E}(\mathcal{Y})$, follows the existence of a subnet $\left\{x_{i(k)}\right\}$ of $\left\{x_{i}\right\}$ such that $x_{i(k)}=a_{k} j_{k}, x_{i(k)} y=b_{k} m_{k}$, where $j_{k}, m_{k} \in J,\left\{a_{k}\right\}$ and $\left\{b_{k}\right\}$ converge to $e$ in $\tau, j_{k} \rightarrow e$ in $\mathscr{U}$, and $m_{k} \rightarrow e$ in $\mathcal{E}(\mathcal{Y})$. Therefore

$$
j_{k}^{-1} m_{k}=y m_{k}^{-1} b_{k}^{-1} a_{k} m_{k} \text {. }
$$

Since we know that $b_{k}^{-1} a_{k} \rightarrow e$ in $\tau$, that $m_{k} \rightarrow e$ in $\mathcal{E}(\mathcal{Y})$, and that Ad is $\mathcal{E}(\mathcal{Y})$-continuous on $L$, we conclude from our observations in $\S 2$ that $\left\{m_{k}^{-1} b_{k}^{-1} a_{k} m_{k}\right\}$ must converge to $e$ in $\tau$. Since $J$ is $\tau$-closed and contains each $j_{k}^{-1} m_{k}$, this implies that $y \in J$ and completes the proof of (iii).

Finally, we prove 3.2(iv). By 3.1, $H=V \times Z(L)$ is decisive in $L$ and Ad maps $V$ injectively into a torus $Q$. We may assume that the basis $\left\{v_{1}, \ldots, v_{n}\right\}$ of $V$ is such that the projection of $\operatorname{Ad}\left(x_{1} v_{1}+\cdots+x_{n} v_{n}\right), x_{m} \in \mathbf{R}$, onto the first $n$ factors of $Q$ is $\left(e^{i x_{1}}, \ldots, e^{i x_{n}}\right)$. Let $Y$ be the subgroup of $V$ generated by $\left\{2 \pi v_{1}, \ldots, 2 \pi v_{n}\right\}$, and let $J=Y \times Z(L)$. If $\mathscr{U} \in T(L, \tau)$, then $\mathcal{U}=\mathcal{E}\left(\mathscr{U}_{H}\right)$ and Ad: $V \times Z(L) \rightarrow Q$ is $\mathcal{Q}_{H}$-continuous by 3.1. It follows easily from the definition of standard extension that $\mathscr{U}_{H}$ is the standard extension to $H$ of $\mathscr{U}_{J}$, and thus $\mathscr{U}=\mathcal{E}\left(\mathscr{U}_{J}\right)$. This completes the proof of Theorem 3.2.

6. An interesting example. As we have already observed, the methods of $\S 4$ provide topologies for abelian Lie groups which, though weaker than the usual topology, nevertheless involve the compact factor in an essential way. From 4.3 and 4.5, for example, we see that the topology of $\mathbf{R} \times T^{1}$ can be weakened in such a way that $\{(n !+1, n \pi \bmod \mathbf{Z})\}$ or $\left\{\left(n !+1, \frac{n}{2} \bmod \mathbf{Z}\right)\right\}$ converges to the identity. Restricting the latter topology to the product of $\mathbf{R}$ and the two-element group, which we will write as $G=\{0,1\}$, we obtain a topology for $\mathbf{R} \times G$ in which $((2 n) !+1,0) \rightarrow(0,0)$, $((2 n+1) !+1,1) \rightarrow(0,0)$, and $((2 n+1) !+1,0) \rightarrow(0,1)$. If we denote by $\mathcal{U}$ and $\mathcal{V}$, respectively, the usual and the weakened topologies for $\mathbf{R} \times G$, it follows that $\mathbf{R} \times G$ is the $\mathfrak{V}$-closure of $\mathbf{R} \times 0$ and is therefore $\mathfrak{V}$-connected. Applying 4.3 and 3.2 of [1] to the second-countable Lie group $\mathbf{R} \times G$, we find that $\mathcal{Q}$ is the locally arcwise-connected topology associated with $\mathcal{V}$ and that $\mathscr{U}$ and $\mathscr{V}$ have the same arcs. Thus $(\mathbf{R} \times G, \mathfrak{V})$ is a connected but not arcwise-connected topological group. Such 
groups are not, of course, unknown (see [4], for instance), but our example seems to be a particularly simple one.

The investigation of such pathologies derives added significance from its relationship to immersions of analytic groups. Let $(G, \mathcal{Q})$ be a topological group, and let $L$ be a $\mathscr{Q}$-dense subgroup of $G$ which is a connected Lie group in a topology $\tau$ that is stronger than $\mathcal{Q}_{L}$. If $H$ is a decisive subgroup of $L$ and if $\bar{H}$ denotes the $थ$-closure of $H$, then $\bar{H}$ completely determines many topological characteristics (local compactness, finite-dimensionality, metrizability, etc.) of $(G, \mathcal{Q})$. These facts, which resemble but go somewhat beyond recent results of Goto ( $\$ 9$ and 11 of [3]), will be proved by the author in a subsequent paper.

\section{REFERENCES}

1. A. Gleason and R. Palais, On a class of transformation groups, Amer. J. Math. 79 (1957), 631-648.

2. M. Goto, Absolutely closed Lie groups, Math. Ann 204 (1973), 337-341.

3. __ Immersions of Lie groups, J. Math. Soc. Japan 32 (1980), 727-749.

4. F. B. Jones, Connected and disconnected plane sets and the functional equation $f(x)+f(y)=f(x+y)$, Bull. Amer. Math. Soc. 49 (1942), 115-120.

5. G. Mostow, The fundamental group of a homogeneous space, Ann. of Math. 66 (1957), 249-255

6. J. Nienhuys, Not locally compact monothetic groups. I, II, Nederl. Akad. Wetensch. Proc. Ser. A 73 = Indag. Math. 32 (1970), 295-326.

7. Construction of group topologies on abelian groups, Fund. Math. 75 (1972), 101-116.

8. T. C. Stevens, Weakening the topology of a Lie group, Trans. Amer. Math. Soc. (to appear).

9. D. Zerling, Some theorems on (CA) analytic groups, Trans. Amer. Math. Soc. 205 (1975), 181-192.

Department of Mathematics, Mount Holyoke College, South Hadley, Massachusetts 01075

Current address: St. Edmund's House, Cambridge CB3 OBN, England 